\title{
Comparison of protein profiles between acetonitrile- and non-acetonitrile-treated sera from patients with nasopharyngeal carcinomas
}

\author{
YUAN-JIAO HUANG ${ }^{*}$, KAI-FENG DENG ${ }^{*}$, CHAO XUAN*, \\ BEI-BEI ZHANG, YI ZHOU, XIAOLI YANG and MING HE \\ Medical Scientific Research Center, Guangxi Medical University, Nanning 530021, P.R. China
}

Received October 28, 2010; Accepted March 2, 2011

DOI: $10.3892 / 01.2011 .284$

\begin{abstract}
Serum proteins may be abnormally increased or decreased during the occurrence and development of nasopharyngeal carcinoma (NPC). However, currently there are no simple or effective methods to collect and differentiate these abnormally secreted proteins from abundant serum proteins. In this study, acetonitrile was used to remove the majority of high-abundance proteins from serum samples obtained from patients with NPC. The samples were subjected to surface-enhanced laser desorption/ionization time-of-flight mass spectrometry with a CM10 (weak cation exchange) ProteinChip, and the resulting protein profiles were compared with those of non-acetonitrile-treated serum samples. The results showed that the protein profiles differed between the acetonitrile- and non-acetonitrile-treated sera from patients with NPC. A large proportion of the non-acetonitrile-treated NPC serum protein peaks were $<6000 \mathrm{kDa}$, while the detection rate of protein peaks $>6000 \mathrm{kDa}$ was relatively higher in the acetonitrile-treated NPC sera, accounting for more than half of all protein peaks $(26.2+37.5 \%)$. Few differentially upregulated proteins were lost, and the peak value density increased after acetonitrile treatment. In conclusion, acetonitrile treatment of serum samples is effective in removing high-abundance macromolecular proteins. Therefore, acetonitrile treatment can be applied for the investigation of serum proteomics and may aid in the identification of differentially expressed proteins.
\end{abstract}

Correspondence to: Dr Yuan-Jiao Huang, Medical Scientific Research Center, Guangxi Medical University, Nanning 530021, P.R. China

E-mail: hyjgxmu@126.com

*Contributed equally

Keywords: acetonitrile,nasopharyngeal carcinoma, surface-enhanced laser desorption/ionization time-of-flight mass spectrometry

\section{Introduction}

Serum often contains abnormally expressed proteins during tumorigenesis (1). Serum proteins may be abnormally increased or decreased during the occurrence and development of nasopharyngeal carcinoma (NPC) (2). However, there are currently no simple or effective methods to collect and differentiate these abnormally secreted marker proteins from the numerous serum proteins. In this study, acetonitrile was used to remove the majority of high-abundance proteins from serum samples obtained from patients with NPC. Surface-enhanced laser desorption/ionization time-of-flight mass spectrometry (SELDI-TOF-MS) with a CM10 (weak cation exchange) ProteinChip was then used to detect proteins, and the results were compared with those for non-acetonitrile-treated NPC sera. These techniques were utilized to better understand the protein profiles of acetonitrile-treated and -non-acetonitriletreated sera from NPC patients and provide a new method for collecting and identifying serum proteins showing abnormal upregulation or downregulation in a specific disease setting.

\section{Materials and methods}

Serum samples. Serum samples for acetonitrile treatment were obtained from patients with NPC who were initially diagnosed at the Otolaryngological Department of our hospital between November 2007 and April 2008. The inclusion criteria were: patients with pathologically confirmed poorly differentiated squamous cell carcinoma naïve to prior treatment and with no other malignant disease. A total of 58 patients with NPC (30 males and 28 females) aged $44.7 \pm 7.9$ years were enrolled in this study. Serum samples were also obtained from healthy controls who received physical examinations at the Physical Examination Center of our hospital in April 2008. Inclusion criteria were: physically healthy individuals without a history of NPC or any other malignant disease. A total of 58 healthy controls (30 males and 28 females) aged $44.0 \pm 10.5$ years were enrolled in the study.

For acetonitrile treatment, serum samples were obtained from patients with NPC who were first diagnosed at our hospital between July 2009 and November 2009. A total of 30 patients with NPC (22 males and 8 females) aged $44.3 \pm 6.8$ years were 
enrolled. Serum samples were also obtained from 30 healthy controls (22 males and 8 females) aged 43.0 \pm 9.2 years who donated blood at Nanning Central Blood Station in January 2010. Four subjects ( 2 males and 2 females) were selected from each group to construct four subgroups (gender-matched patients and healthy controls per subgroup) for ProteinChip screening. The serum samples were voluntarily donated, and all subjects provided written informed consent.

ProteinChip, mass spectrometer and reagents. The CM10 ProteinChip fromBio-Rad (FosterCity,CA,USA) was used with a ProteinChip Biomarker System. Data were analyzed using ProteinChip 3.2 and Biomarker Wizard software (Ciphergen Biosystems, Freemont, CA, USA). The reagents included U9 buffer (9 mol/l urea and 2\% CHAPS) supplemented with $0.1 \%$ DTT, fresh saturated sinapinic acid (SPA) solution (50\% acetonitrile $+0.5 \%$ TFA) and CM10 binding buffer (50 mM NaAC, $\mathrm{pH} 4.0$ ).

Preparation of non-acetonitrile-treated serum samples. Venous blood samples were collected and left to stand for $30 \mathrm{~min}$ to $1 \mathrm{~h}$. Samples were then centrifuged at $3000 \mathrm{rpm}$ at $4^{\circ} \mathrm{C}$ for $10 \mathrm{~min}$. Non-hemolytic serum was collected, divided into aliquots, and stored at $-80^{\circ} \mathrm{C}$. Before use, the serum was centrifuged at $10,000 \mathrm{rpm}$ at $4^{\circ} \mathrm{C}$ for $2 \mathrm{~min}$. Each chip spot required $10 \mu \mathrm{l}$ of serum. The serum was diluted with 2 volumes of U9 buffer, agitated in an ice bath for $30 \mathrm{~min}$, and $360 \mu \mathrm{l}$ of the relevant binding buffer was added, diluted 40 times and mixed. Then, $100 \mu \mathrm{l}$ was retrieved for loading on pre-treated chips.

Preparation of acetonitrile-treated serum samples. Serum samples were collected and stored as described above. Then, 1.2 volumes of acetonitrile was added to $200 \mu \mathrm{l}$ of serum, vortexed, and left to stand at $4^{\circ} \mathrm{C}$ for $10 \mathrm{~min}$. Following centrifugation at $12,000 \mathrm{rpm}$ for $35 \mathrm{~min}$, the supernatant was retained and stored at $-80^{\circ} \mathrm{C}$ until use. To prepare each sample for use, $40 \mu \mathrm{l}$ of $0.9 \%$ normal saline was added and $10 \mu \mathrm{l}$ of the protein solution was collected and treated as described for the preparation of non-acetonitrile-treated samples.

Pre-treatment of the ProteinChip. The ProteinChip array cassette was placed in the bioprocessor, and $200 \mu 1$ of binding solution was added to each well. The ProteinChip was incubated for $5 \mathrm{~min}$ at room temperature and agitated vigorously. This was repeated once, and the ProteinChip was kept moist until use.

Preparation of the ProteinChip. The ProteinChip was prepared in accordance with the manufacturer's instructions. Following preparation of the serum samples and pre-treatment of the ProteinChip, as described above, $100 \mu 1$ of sample was added to each well. The ProteinChip was agitated at $400-600 \mathrm{rpm}$ at $4^{\circ} \mathrm{C}$ for $2 \mathrm{~h}$, washed with $200 \mu 1$ of deionized water, and then rapidly spun dry. Finally, $0.5 \mu 1$ of SPA was added to each well twice, and proteins were detected after the ProteinChip had dried.

Protein detection. Proteins in the acetonitrile- and nonacetonitrile-treated serum samples were detected using the ProteinChip Biomarker System MS. Data were collected by

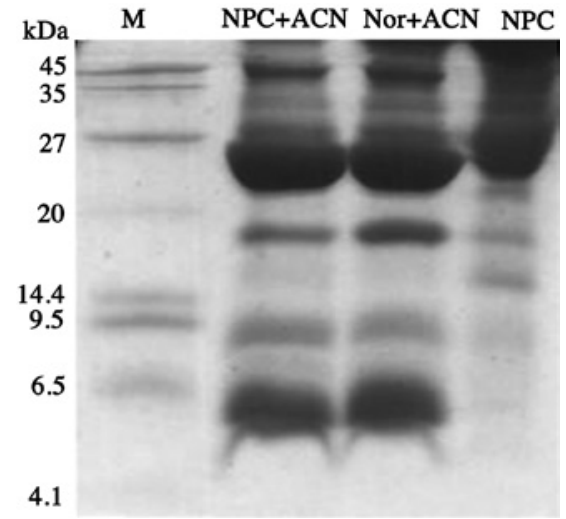

Figure 1. Smaller molecular proteins in serum were enriched by acetonitrile precipitation. M, molecular marker; NPC+ACN, serum of nasopharyngeal carcinoma + acetonitrile; Nor+ACN, normal serum + acetonitrile; NPC, serum of nasopharyngeal carcinoma.

averaging the results of 130 laser shots with an intensity of 180 , a detector sensitivity of 8 , data collection range of $\mathrm{m} / \mathrm{z}$ 2,000-50,000, and an optimization range of $\mathrm{m} / \mathrm{z} 2,000-10,000$. Data signals were collected 20 times per spot.

Normalization, collection and analysis of protein profiles. Mass spectrograms from the same chip were combined and normalized using ProteinChip Software version 3.2. The range of peak masses was analyzed between m/z 2,000 and 50,000. Peak detection was performed using Biomarker Wizard software. The settings for 'auto-detect peaks to cluster' were: the signal-to-noise ratio was 5 and the minimum peak threshold was $15 \%$ for the first pass. For cluster completion, the cluster mass window was $0.3 \%$, and the signal-to-noise ratio for the second pass was 2 . Finally, the protein profiles were obtained using Biomarker Wizard software. The number of peaks in the spectra and the intensity of each peak were recorded and analyzed in the acetonitrile- and non-acetonitrile-treated serum samples.

\section{Results}

Small molecular proteins in serum were enriched by acetonitrile precipitation. As shown in Fig. 1, after precipitating $200 \mu$ of serum with 1.2 volumes of acetonitrile, Tricine-SDSPAGE electrophoresis (3) indicated that proteins/polypeptides $<30 \mathrm{kDa}$ were enriched.

Protein profiles of acetonitrile- and non-acetonitrile-treated sera from patients with NPC and healthy controls. MS protein profiles were obtained for the acetonitrile- and nonacetonitrile-treated sera from patients with NPC or healthy controls using the CM10 ProteinChip (Fig. 2). Statistical analysis of the protein profiles for the non-acetonitriletreated NPC patient sera $(n=58)$ and normal healthy control sera $(n=58)$ using the conditions described in Materials and methods revealed a total of 99 protein or polypeptide peaks in the non-acetonitrile-treated NPC sera, with a detection rate of $18.1 \%$ for proteins with a molecular weight (MW) of 6,000-10,000 kDa, $11.1 \%$ for proteins $>10,000 \mathrm{kDa}$, and $5.1 \%$ for proteins at a peak density of $>5$. In contrast, 80 protein or 


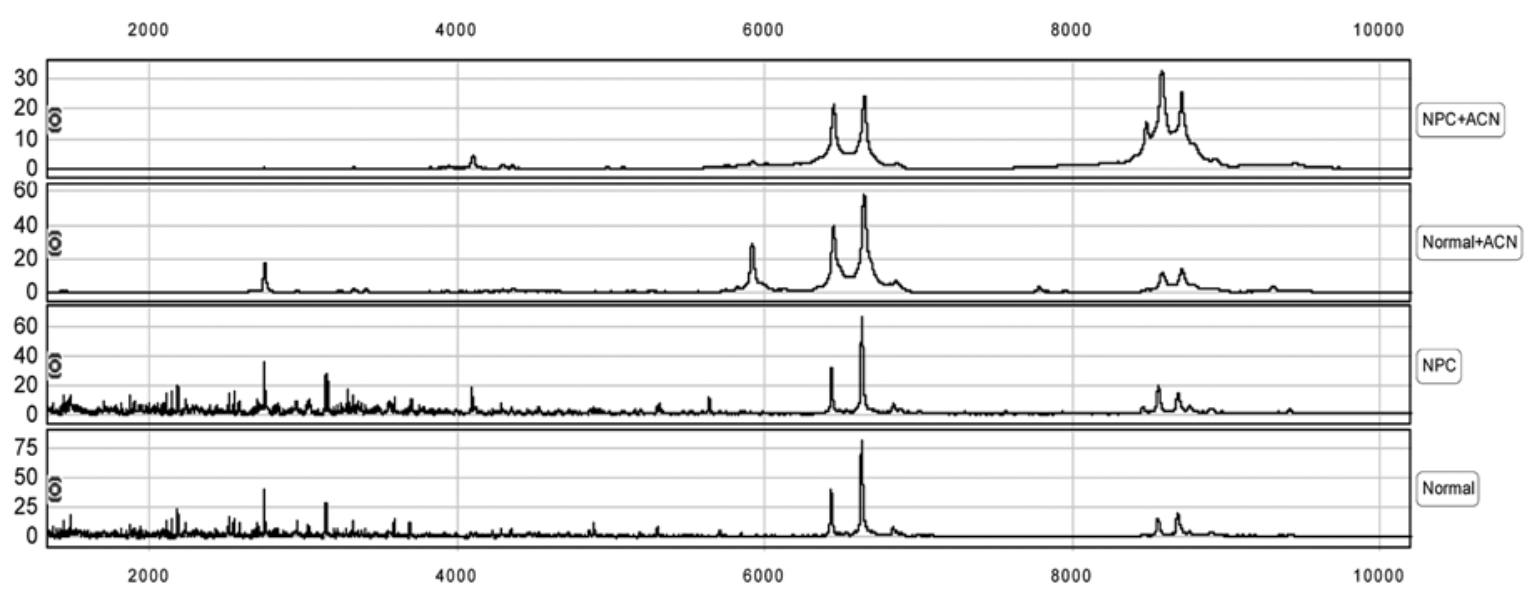

Figure 2. Serum protein (2,000-10,000 kDa) MS of acetonitrile- and non-acetonitrile-treated serum samples from patients with NPC or healthy controls. The relative intensity is provided for each peak with one $\mathrm{H}^{+}$. NPC, nasopharyngeal carcinoma; ACN, acetonitrile; normal, normal serum; peak 1: $5638.8 \mathrm{kDa}$ $(\mathrm{NPC}+\mathrm{ACN})$ or $5633.1 \mathrm{kDa}(\mathrm{NPC})$; peak 2: $8480.3 \mathrm{kDa}, 8579.0 \mathrm{kDa}(\mathrm{NPC}+\mathrm{ACN})$ or $8563.9 \mathrm{kDa}(\mathrm{NPC})$ and $8705.1 \mathrm{kDa}(\mathrm{NPC}+\mathrm{ACN})$ or $8691.8 \mathrm{kDa}(\mathrm{NPC})$.

Table I. Detection of proteins using a CM10 ProteinChip in acetonitrile- and non-acetonitrile-treated sera from patients with NPC or healthy controls.

\begin{tabular}{|c|c|c|c|c|c|c|c|c|c|c|c|c|c|c|}
\hline \multirow{3}{*}{ MW range $(\mathrm{kDa})$} & \multirow{3}{*}{$\begin{array}{l}\text { Peaks } \\
\text { (n) }\end{array}$} & \multicolumn{6}{|c|}{ CM10 } & \multirow{3}{*}{$\begin{array}{c}\text { Peaks } \\
\text { (n) }\end{array}$} & \multicolumn{6}{|c|}{$\mathrm{CM} 10+\mathrm{ACN}$} \\
\hline & & \multicolumn{3}{|c|}{ NPC intensity } & \multicolumn{3}{|c|}{ Normal intensity } & & \multicolumn{3}{|c|}{ NPC intensity } & \multicolumn{3}{|c|}{ Normal intensity } \\
\hline & & $<5$ & $5-10$ & $>10$ & $<5$ & $5-10$ & $>10$ & & $<5$ & $5-10$ & $>10$ & $<5$ & $5-10$ & $>10$ \\
\hline 2,000 & 23 & 22 & 0 & 1 & 21 & 1 & 1 & 4 & 3 & 1 & 0 & 3 & 0 & 1 \\
\hline 3,000 & 19 & 19 & 0 & 0 & 19 & 0 & 0 & 9 & 9 & 0 & 0 & 9 & 0 & 0 \\
\hline 4,000 & 15 & 14 & 0 & 1 & 13 & 2 & 0 & 9 & 8 & 0 & 1 & 9 & 0 & 0 \\
\hline 5,000 & 13 & 12 & 1 & 0 & 11 & 2 & 0 & 7 & 6 & 0 & 1 & 6 & 0 & 1 \\
\hline 6,000 & 5 & 3 & 1 & 1 & 3 & 1 & 1 & 6 & 4 & 0 & 2 & 4 & 0 & 2 \\
\hline 7,000 & 3 & 3 & 0 & 0 & 3 & 0 & 0 & 4 & 4 & 0 & 0 & 3 & 1 & 0 \\
\hline 8,000 & 6 & 6 & 0 & 0 & 6 & 0 & 0 & 6 & 1 & 2 & 3 & 3 & 0 & 3 \\
\hline 9,000 & 4 & 4 & 0 & 0 & 4 & 0 & 0 & 5 & 4 & 1 & 0 & 4 & 1 & 0 \\
\hline 10,000 & 5 & 5 & 0 & 0 & 5 & 0 & 0 & 16 & 16 & 0 & 0 & 16 & 0 & 0 \\
\hline 20,000 & 6 & 6 & 0 & 0 & 6 & 0 & 0 & 14 & 14 & 0 & 0 & 14 & 0 & 0 \\
\hline Total & 99 & 94 & 2 & 3 & 91 & 6 & 2 & 80 & 69 & 4 & 7 & 71 & 2 & 7 \\
\hline$\%$ of $6,000-10,000$ & 18.1 & & & & & & & 26.3 & & & & & & \\
\hline$\%$ of $>10,000$ & 11.1 & & & & & & & 37.5 & & & & & & \\
\hline$\%$ of $>5$ intensity & 5.1 & & & & & & & 13.8 & & & & & & \\
\hline
\end{tabular}

polypeptide peaks were detected in the acetonitrile-treated NPC sera, with a detection rate of $26.2 \%$ for proteins with a MW of 6,000-10,000 kDa, 37.5\% for proteins with a MW of $>10,000 \mathrm{kDa}$, and $13.8 \%$ for proteins at a peak density of $>5$. Notably, a large proportion of peaks in the non-acetonitriletreated NPC sera were $<6,000 \mathrm{kDa}$, while the detection rate of peaks $>6,000 \mathrm{kDa}$ was higher in the acetonitrile-treated NPC sera, accounting for over half of the peaks $(26.2+37.5 \%)$ (Table I).

Effects of acetonitrile treatment on serum protein profiles of patients with NPC and healthy controls. Statistical analysis of the protein profiles of the non-acetonitrile-treated sera between NPC patients and healthy controls showed that CM10 detected 28 differentially expressed protein peaks at $\mathrm{P}<0.05$ and mean $>\mathrm{SD}$, of which 6 (5633.1, 8563.9, 8691.8, $8765.1,8901.9$ and $13738.6 \mathrm{kDa})$ were upregulated, and 22 were downregulated (Fig. 3). A comparison of the acetonitrile-treated sera between NPC patients and healthy controls showed that CM10 detected 13 differentially expressed protein peaks at $\mathrm{P}<0.05$ and mean $>\mathrm{SD}$, of which 5 (5638.8, $8480.3,8579.0,8705.1$ and $13,772.8 \mathrm{kDa})$ were upregulated, and 8 were downregulated (Fig. 4).

The MWs of the upregulated protein peaks (5633.1, 8563.9, 8691.8 and $13738.6 \mathrm{kDa}$ ) found in the protein profiles of the non-acetonitrile-treated NPC sera were similar to those of 


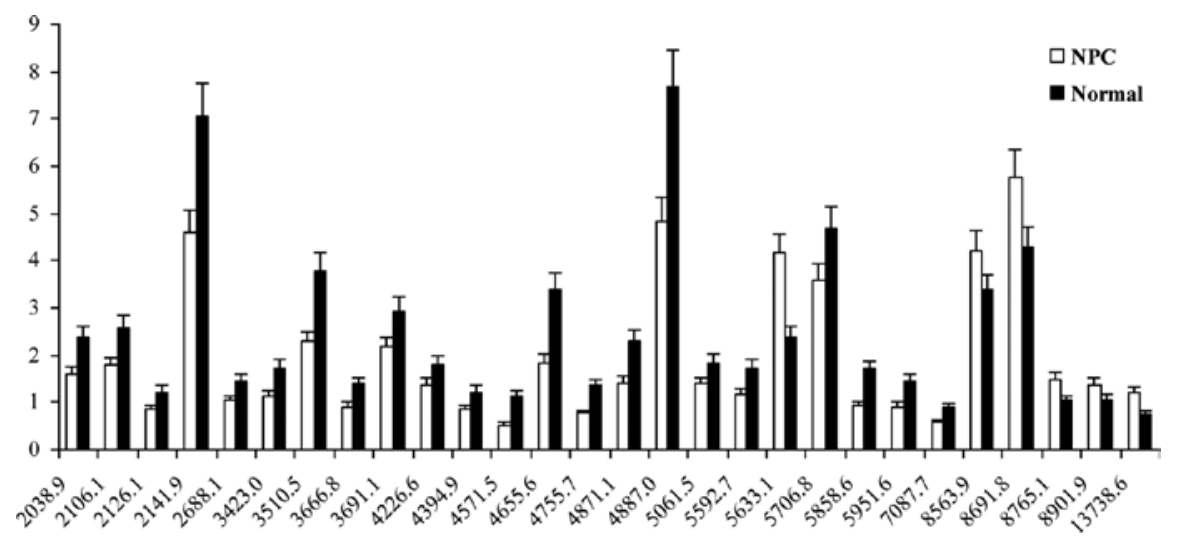

Figure 3. Proteins detected using a CM10 ProteinChip in non-acetonitrile-treated sera from patients with NPC and healthy controls (Normal). Of the 28 differentially expressed proteins, 6 were upregulated and 22 were downregulated. The white and black bars are the serum proteins in the NPC patients and healthy controls, respectively.

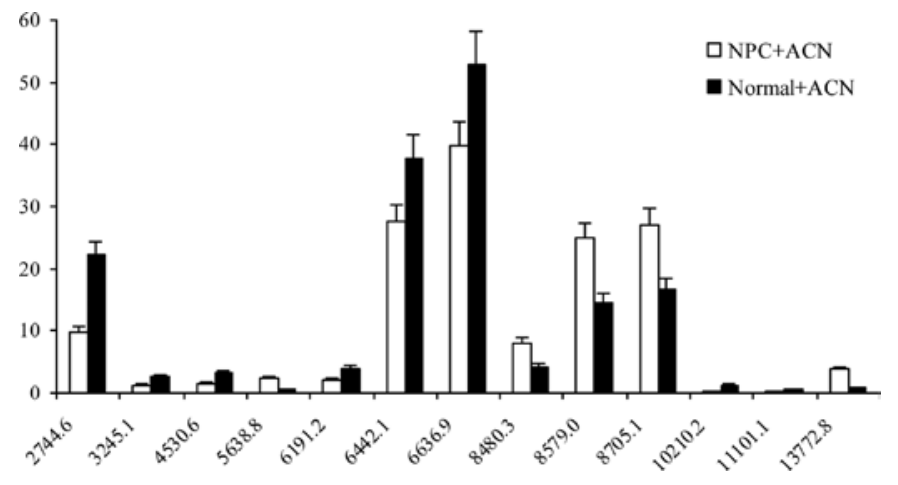

Figure 4. Proteins detected using a CM10 ProteinChip in acetonitriletreated sera from patients with NPC (NPC+ACN) and healthy controls (Normal+ACN). Of 13 differentially expressed proteins, 5 proteins were upregulated and 8 downregulated. The white and black bars are the serum proteins in NPC patients and healthy controls, respectively.

the acetonitrile-treated NPC sera $(5638.8,8579.0,8705.1$ and $13,772.8 \mathrm{kDa}$ ). Specifically, the patterns for 2 protein peaks (8563.9 and $8691.8 \mathrm{kDa}$ ) in the non-acetonitrile-treated NPC sera were the same as those in the acetonitrile-treated NPC sera (8579.0 and $8705.1 \mathrm{kDa})$, although the peak intensity was 5-7 times higher in the latter (Figs. 2-4). Since the equipment and methodology utilized were the same in all of the tests, the four upregulated proteins obtained from the acetonitrileand non-acetonitrile-treated NPC sera can be regarded as the same proteins/polypeptide.

\section{Discussion}

Proteins perform a wide range of physiological functions, and the existence of life is dependent on protein activity. Pathophysiological changes and drug effects are closely related to changes in the components or quantities of proteins. Human serum contains many low-abundance proteins secreted by and shed from tissue cells. These proteins may be important proteins for signal conduction and regulation. Furthermore, necrosis, apoptosis, hemolysis and tumorigenesis may cause intracellular proteins to be released into the blood $(1,4)$. Serum proteomics research focusing on low-abundance proteins in NPC may aid in both the identification of protein markers for NPC and a better understand of its pathogenesis, and thus reveal new techniques for the diagnosis of NPC. Furthermore, such proteins may offer potential targets for treatment or serve as markers that monitor disease course and treatment effectiveness.

Each milliliter of serum contains $60-80 \mathrm{mg}$ of protein, mainly albumin (50-70\%), immunoglobulins ( $\operatorname{IgG}, \operatorname{IgA}, \operatorname{IgD}$, $\operatorname{IgM}$, and $\operatorname{IgE}$; IgG accounts for $10-25 \%$ of the total protein content), transferrin, haptoglobin, and lipoprotein. The majority of the remaining proteins are of low abundance (5). When using SELDI-TOF-MS to analyze serum proteins, highabundance albumin and $\operatorname{IgG}$ can interfere with the detection of low-abundance proteins, thereby affecting the resulting profiles (6).

Acetonitrile can decrease the specific inductivity of water, and biomacromolecules with hydrophilic surfaces may be dehydrated causing them to aggregate and ultimately precipitate. Accordingly, acetonitrile treatment can precipitate and remove many high-abundance proteins from serum and eliminate the intra-molecular interactions between proteins. Acetonitrile can also degenerate and disassociate low-abundance proteins/polypeptides that initially bind to high-abundance proteins such as albumin. As a result, these low-abundance proteins or polypeptides are not removed when eliminating the high-abundance macromolecules (7). In this study, acetonitrile was used to treat serum samples obtained from NPC patients to remove the high-abundance macromolecular proteins, and the protein profiles were then compared with non-acetonitrile-treated NPC sera using SELDI-TOF-MS technology with a CM10 ProteinChip. Under standard conditions, 99 protein or polypeptide peaks were detected in the non-acetonitrile-treated sera from the NPC patients, with a detection rate of $18.1 \%$ for proteins with a MW of $6,000-10,000 \mathrm{kDa}, 11.1 \%$ for proteins with a MW of $>10,000 \mathrm{kDa}$, and $5.1 \%$ for proteins at a peak density of $>5$. In contrast, 80 peaks were detected in the acetonitrile-treated sera from the NPC patients, with a detection rate of $26.2 \%$ for proteins with a MW of $6,000-10,000 \mathrm{kDa}, 37.5 \%$ for proteins with a MW of $>10,000 \mathrm{kDa}$, and $13.8 \%$ for proteins at a peak density of $>5$. Compared with non-acetonitrile-treated sera from healthy subjects, 28 differentially expressed protein peaks were detected in the non-acetonitrile-treated sera from 
NPC patients $(\mathrm{P}<0.05$; mean $>\mathrm{SD})$, of which 6 peaks were upregulated and 22 were downregulated. In comparison, for acetonitrile-treated sera, 13 peaks were differentially expressed in patients with NPC versus the healthy controls $(\mathrm{P}<0.05$; mean $>\mathrm{SD})$, of which 5 peaks were upregulated and 8 were downregulated in NPC patients. Four differentially expressed proteins were upregulated in the acetonitrile- and non-acetonitrile-treated NPC sera compared with the healthy controls, although the latter had lower peak values, suggesting these proteins are promising markers for NPC. However, the downregulated proteins differed between the acetonitrile- and non-acetonitrile-treated NPC sera profiles.

Using SELDI-TOF-MS in conjunction with a CM10 ProteinChip, striking differences were noted in the protein profiles of the acetonitrile- and non-acetonitrile-treated NPC sera. Of note is that a large proportion of the proteins from the non-acetonitrile-treated NPC sera were $<6,000 \mathrm{kDa}$, while the detection rate of protein peaks $>6,000 \mathrm{kDa}$ was higher in the acetonitrile-treated NPC sera, accounting for over half of all protein peaks detected $(26.2+37.5 \%)$. Four upregulated proteins were found in both the acetonitrile- and non-acetonitriletreated sera from the NPC patients; these 4 proteins may be valuable markers for NPC. Few upregulated proteins were lost, and the peak value densities increased by 5-7-fold following acetonitrile treatment. Therefore, acetonitrile is effective in eradicating the majority of the high-abundance macromolecular proteins from serum samples of patients with NPC. Acetonitrile treatment can be applied in serum proteomics research and may facilitate the detection and identification of significant differentially expressed proteins.

\section{Acknowledgements}

This study was supported by the Local High Disease Control and Prevention Research Laboratory Foundation of Guangxi, China (no. 0630006-5E7Z; no. 0842009-Z14) and The Natural Science Foundation of Guangxi, China (no. 06390-18).

\section{References}

1. Adkins JN, Varnum SM, Auberry KJ, et al: Toward a human blood serum proteome: analysis by multidimensional separation coupled with mass spectrometry. Mol Cell Proteomics 1: 947-955, 2002.

2. Huang YJ, Xuan C, Zhan BB, et al: SELDI-TOF MS profiling of serum for detection of nasopharyngeal carcinoma. J Exp Clin Cancer Res 28: 85, 2009.

3. Schägger H: Tricine-SDS-PAGE. Nat Protoc 1: 16-22, 2006.

4. Ma PC, Blaszkowsky L, Bharti A, et al: Circulating tumor cells and serum tumor biomarkers in small cell lung cancer. Anticancer Res 23: 49-62, 2003.

5. Steel LF, Trotter MG, Nakajima PB, et al: Efficient and specific removal of albumin from human serum samples. Mol Cell Proteomics 2: 267-270, 2003.

6. Li J, Zhang Z, Rosenzweig J, et al: Proteomics and bioinformatics approaches for identification of serum biomarkers to detect breast cancer. Clin Chem 48: 1296-1304, 2002.

7. Chertov O, Biragyn A, Kwak LW, et al: Organic solvent extraction of proteins and peptides from serum as an effective sample preparation for detection and identification of biomarkers by mass spectrometry. Proteomics 4: 1195-1203, 2004. 